\title{
Spatially patterned gradients of synaptic connectivity are established early in the developing retina Susana Cohen-Cory
}

Address: Department of Neurobiology and Behavior, University of California, Irvine, 2205 McGaugh Hall, Irvine, CA 92697, USA. Email: scohenco@uci.edu

Published: 9 June 2008

Journal of Biology 2008, 7:15 (doi:10.1 186/jbiol76)

The electronic version of this article is the complete one and can be found online at http://jbiol.com/content/7/5/I5

(c) 2008 BioMed Central Ltd

\begin{abstract}
Retinal neurons receive input from other cells via synapses and the position of these synapses on the neurons reflects the retinal regions from which information is received. A new study in Neural Development establishes that the spatial distribution of excitatory synaptic inputs emerges at the onset of synapse formation rather than as a result of changes during neuronal reorganisation.
\end{abstract}

How do appropriate synaptic patterns of connectivity emerge during development? What is the relationship between a neuron's dendritic architecture and its synaptic connectivity? These questions have puzzled developmental neurobiologists for many years, but only recently, with the advent of novel neuronal labeling and imaging techniques, have answers to these questions been obtained in the living organism. In a recent article in Neural Development, Morgan et al. [1] investigate the developmental mechanisms by which excitatory synaptic inputs become distributed across retinal neurons so that reliable visual information can be transferred to the brain. This study demonstrates that synaptic inputs to retinal projection neurons are spatially patterned from early stages of dendritic development and that the density of inputs is maintained as constant, even as the retinal circuits remodel and mature.

The organized laminated structure of the vertebrate retina (Figure 1a) provides an excellent model in which to study how synaptic circuits are established during development and to what extent intrinsic versus extrinsic signals contribute to this process. Synaptic circuits in the retina transform visual information that is collected by photoreceptors into electrical and chemical signals, which are then transferred to retinal ganglion cells (RGCs), the output neurons of the retina. RGCs relay visual information to the brain through their long projecting axons. Those RGCs, whose cell bodies reside in the ganglion cell layer, receive synaptic input onto their dendrites in the inner plexiform layer in the form of excitatory and inhibitory synapses from bipolar cells and amacrine cells. The inhibitory synapses signal using $\gamma$ aminobutyric acid (GABA), whereas the excitatory synapses signal using glutamate. The dendrites of RGCs are remodeled extensively during development: initially as the first inhibitory GABAergic synapses between amacrine cells and RGCs are formed, and then in response to the first excitatory glutamatergic inputs from bipolar cells to RGCs [2,3]. This dynamic remodeling of dendritic arbors [4] works together with molecular cues [5] to organize inputs into different sublaminae in the inner plexiform layer in response to visual signals. In this way, the distinct types of RGCs attain their characteristic dendritic lamination, architecture and synaptic connectivity.

Evidence of dynamic mechanisms of synaptogenesis and their relation to dendritic arbor structure has been obtained in recent studies that have expressed fluorescently tagged postsynaptic components in individual neurons in live fish, 


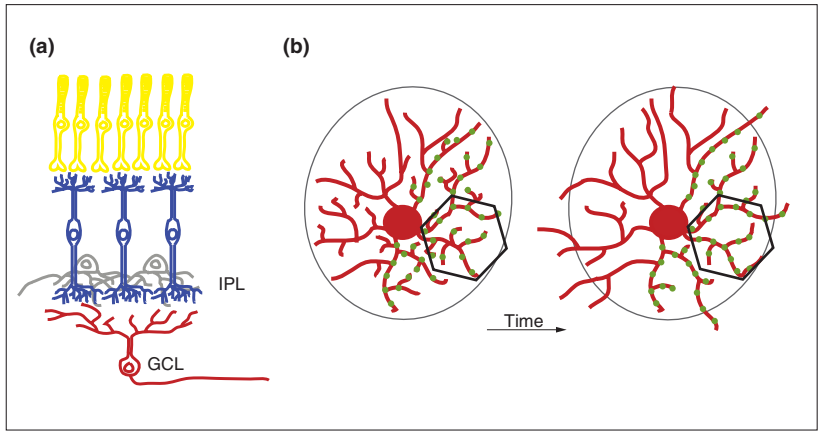

Figure I

Retinal connections and their remodeling. (a) Simplified diagram of a retinal circuit, illustrating the organization of inputs from photoreceptor to bipolar to retinal ganglion cells (RGCs). Photoreceptors (yellow) transfer visual information to bipolar cells (blue) that in turn contact dendrites of RGCs (red). Amacrine cells (gray) also provide synaptic input to RGCs. (b) Schematic representation of the relationship between dendritic architecture and synaptic connectivity of a developing RGC. Excitatory synaptic sites on RGC dendrites can be visualized by the punctate distribution of PSD95-YFP (green) on neurons expressing a red fluorescent protein (red). During development, the dendritic arbor of an RGC extends its synaptic territory (oval) through dynamic remodeling of its branches. The number of synaptic contacts per unit area of the bipolar cell surface (represented by the hexagon) remains constant as dendrites remodel and exuberant branches are pruned back. Synapse density is maintained by an increase in the number and/or density of synapses on those branches that are retained. For visual clarity, synaptic sites are illustrated only in a portion of the dendritic arbor. GCL, ganglion cell layer; IPL, inner plexiform layer.

frog and mouse embryos [6-8]. The postsynaptic density protein PSD-95 is a scaffolding protein that participates in synapse maturation and has served as a marker for glutamatergic postsynaptic sites in vivo [9]. In their new study, Morgan and colleagues [1] show a new correlation of the emergence of glutamatergic synaptic inputs on RGCs with their dendritic arbor structure by analyzing RGCs expressing PSD-95 tagged with yellow fluorescent protein (YFP) at key stages in retinal circuit development. They developed a set of elegant measuring tools to examine the density and distribution of putative glutamatergic synaptic sites on RGC dendrites (bipolar cell inputs) in explanted mouse retinas, from postnatal day 5, before functional glutamatergic responses are recorded, until the first postnatal month, when functional circuits are mature. They focused on monostratified and ON and OFF bistratified RGCs to determine whether the distinct spatial patterns of bipolar cell inputs are established at the onset of synaptogenesis or whether they emerge through a remodeling process. Monostratified RGCs (ON-center or OFF-center) position their dendrites in either one of two sublaminae to receive functional input from ON or OFF visual pathways. Bistratified RGCs have dendrites that stratify or branch in both sublaminae and receive inputs from both $\mathrm{ON}$ and OFF visual pathways.

Confirming previous observations that chimeric PSD-95 expression localizes to ultrastructurally identified synapses in vivo [7], the authors [1] found that expression of PSD95YFP on RGC dendrites localized to sites where bipolar cell synapses form. Synaptic sites were found to be evenly distributed along the RGC dendritic arbor (which was visualized by the expression of the red fluorescent protein td-Tomato) before synaptic glutamate responses could be recorded, and the patterned distribution of synaptic sites on individual branches was maintained as the dendritic arbor stratified and remodeled. A centro-peripheral gradient of synaptic number, with more synapses closer to the cell body, emerges early, when bipolar cells are forming synapses onto RGCs, and this gradient is maintained despite ongoing remodeling and synaptogenesis. It is likely that centroperipheral synapse gradients are established by competition between bipolar inputs at the borders of the dendritic arbor, where the border of an RGC receptive field locates. Live imaging of synaptic sites on distal branches [3] could now be used to demonstrate the existence of a dynamic competitive process at receptive field borders.

Using a tool that measures the lamination index of the dendritic branches and of synaptic sites, Morgan et al. [1] also revealed that the dendritic arbors of RGCs begin to stratify both their branches and their synapses at the time when synaptic glutamate neurotransmission begins (postnatal days 7-12 in the mouse). During this period, when the dendritic arbor actively enlarges and also refines by pruning back dendrites, the number of synaptic contacts per unit area of the bipolar cell surface is maintained by the increase in synapse density on those branches that are maintained (Figure 1b). Thus, as the retinal circuit matures, dendritic arbors refine but the density of inputs per bipolar cell is retained by the increase in the density of synapses on the dendrite. A constant distribution of bipolar cell synapses on the dendritic membrane of RGCs may be determined by a limitation on the number of synaptic contacts a bipolar cell can make, and this limitation would assure that information is reliably transmitted as the neurons mature. The spatially patterned distribution of excitatory synapses observed as the retina matures has recently been suggested to be responsible for setting the sensitivity of different RGC types to visual stimuli [10], and as demonstrated in this study [1], it is directly related to the density, extent and stratification of the dendritic branches.

The well-controlled, detailed analysis of synapse distribution, growth and stratification of sample RGC dendritic arbors at different stages of development performed by 
Morgan et al. [1] also suggests that branches that are eliminated or pruned back during arbor refinement bear synaptic contacts, and these branches can be those that do make contact with presynaptic bipolar cells. This notion is in agreement with observations that have been made in studies that have followed pre- and postsynaptic components in individual branches by time-lapse imaging of axons and dendrites in vivo [6,11-13]. These studies indicate that RGCs, like other central neurons, transition from an exploratory state to a mature state by removing transient contacts made by excess dendritic branches. Dynamic mechanisms of synaptic and dendritic arbor remodeling seem to be similar for RGCs that maintain their stratification order (dendrites of ON and OFF bistratified RGCs) and those that transition from an immature bistratified to a monostratified dendritic arbor. Time-lapse imaging studies could now be used to reveal the sequence of events by which spatially patterned gradients of synaptic connectivity are established and refined in the retinal circuit, and to ascertain the contribution of early inhibitory and late excitatory inputs to this dynamic process.

In summary, the elegant study by Morgan and colleagues [1] provides new insights into the mechanisms that shape the functional receptive field of an RGC and this contributes to our understanding of the cellular and molecular mechanisms that control synaptic connectivity in the developing retina. It is known that intercellular communication between RGCs and their presynaptic neurons (amacrine and bipolar cells), in the form of activity-dependent as well as molecular signals, is responsible for the remodeling and shape of dendritic arbors. Stratification and laminar refinement are mediated by interactions between transmembrane recognition molecules that are conserved through evolution and that guide the branching patterns of distinct neuronal subtypes [5]. Laminar and synaptic refinement is also modulated by activity-dependent signals, which in turn may control the expression and function of important proteins, such as brain-derived neurotrophic factor (BDNF), within the local retinal circuitry. BDNF contributes to RGC dendritic branching and laminar refinement, acting both locally within the retina and also through retrograde mechanisms acting at the axonal target(s) [14-17]. To what extent these and other local molecular signals contribute to the patterned distribution of excitatory synaptic inputs in the retinal circuit, and how this pattern is modified by activity and potential retrograde signals from the brain, is now open to new investigation. Studies that employ both static and dynamic analyses of synaptic components in living neurons in their natural setting are beginning to provide a long-sought window into the developing brain.

\section{Acknowledgements}

The author is supported by grants from the National Eye Institute (EY$0 I 1912$ ) and the March of Dimes Foundation.

\section{References}

I. Morgan JL, Schubert T, Wong RO: Developmental patterning of glutamatergic synapses onto retinal ganglion cells. Neural Develop 2008, 3:8.

2. Wong WT, Faulkner-Jones BE, Sanes JR, Wong RO: Rapid dendritic remodeling in the developing retina: dependence on neurotransmission and reciprocal regulation by Rac and Rho. J Neurosci 2000, 20:5024-5036.

3. Wong WT, Wong RO: Changing specificity of neurotransmitter regulation of rapid dendritic remodeling during synaptogenesis. Nat Neurosci 200I, 4:35I-352.

4. Tian N, Copenhagen DR: Visual stimulation is required for refinement of ON and OFF pathways in postnatal retina. Neuron 2003, 39:85-96.

5. Yamagata M, Sanes JR: Dscam and Sidekick proteins direct laminaspecific synaptic connections in vertebrate retina. Nature 2008, 45I:465-469.

6. Niell CM, Meyer MP, Smith SJ: In vivo imaging of synapse formation on a growing dendritic arbor. Nat Neurosci 2004, 7:254-260.

7. Sanchez AL, Matthews BJ, Meynard MM, Hu B, Javed S, CohenCory S: BDNF increases synapse density in dendrites of developing tectal neurons in vivo. Development 2006, 133:2477-2486.

8. Gray NW, Weimer RM, Bureau I, Svoboda K: Rapid redistribution of synaptic PSD-95 in the neocortex in vivo. PLoS Biol 2006, 4:e370.

9. Okabe S, Miwa A, Okado H: Spine formation and correlated assembly of presynaptic and postsynaptic molecules. J Neurosci 2001, 21:6105-61 14.

10. Xu Y, Vasudeva V, Vardi N, Sterling P, Freed MA: Different types of ganglion cell share a synaptic pattern. J Comp Neurol 2008, 507:1871-1878.

II. Hu B, Nikolakopoulou AM, Cohen-Cory S: BDNF stabilizes synapses and maintains the structural complexity of optic axons in vivo. Development 2005, I32:4285-4298.

12. Ruthazer ES, Li J, Cline HT: Stabilization of axon branch dynamics by synaptic maturation. J Neurosci 2006, 26:3594-3603.

13. Meyer MP, Smith SJ: Evidence from in vivo imaging that synaptogenesis guides the growth and branching of axonal arbors by two distinct mechanisms. J Neurosci 2006, 26:3604-36I4.

14. Lom B, Cogen J, Sanchez AL, Vu T, Cohen-Cory S: Local and target-derived brain-derived neurotrophic factor exert opposing effects on the dendritic arborization of retinal ganglion cells in vivo. J Neurosci 2002, 22:7639-7649.

15. Liu X, Grishanin RN, Tolwani RJ, Renteria RC, Xu B, Reichardt LF, Copenhagen DR: Brain-derived neurotrophic factor and TrkB modulate visual experience-dependent refinement of neuronal pathways in retina. J Neurosci 2007, 27:7256-7267.

16. Landi S, Cenni MC, Maffei L, Berardi N: Environmental enrichment effects on development of retinal ganglion cell dendritic stratification require retinal BDNF. PLOS ONE 2007, 2: 346.

17. Du JL, Poo MM: Rapid BDNF-induced retrograde synaptic modification in a developing retinotectal system. Nature 2004, 429:878883. 